\title{
Histological Study of Emerging Role of Exosomes Based Therapeutic in Management of Hepatocellular Carcinoma Rat Model
}

Suhaib Naeem ${ }^{1 *}$, Hassan Sabry ${ }^{1}$, Mohammed Abduh ${ }^{1}$, and Marwa Matboly ${ }^{2}$

Departments of ${ }^{1}$ Histology and Cell Biology, Faculty of medicine,Al-Azhar university, Cairo, Egypt and ${ }^{2}$ Medical Biochemistry and Molecular Biology, Faculty of Medicine, Ain Shams University, Cairo, Egypt

*Corresponding author: Suhaib Naeem, Phone: +201004488927, E-Mail: Suhaibnaeem9@ gmail.com and

Suhaibnaeem@azhar.edu.eg

\begin{abstract}
Background: hepatocellular carcinoma (HCC) is the most common type of primary liver cancers with high incidence and mortality rates. The major risk factor for HCC is cirrhosis, which occurs usually due to HBV and HCV infection. Exosomes thought that have a major role on the promotion and spread of HCC, which can be uased as therapeutic and diagnostic marker. Objectives: we aimed to use evaluate their role in HCC assessment and their use as potential selected diagnostic biomarkers, and to characterize the efficacy of Hesperidin on modulating exosomal production and exosomal non-coding RNA related HCC expression using animal models.

Materials and Methods: the study included 30 Rats devised to 5 groups 6 on each, controle and DiethyleNitros amine (DEN) and Acetamidofluorene (2-AAF) to iduce HCC group and Hispiridine treated groups with three different dosos $50 \mathrm{mg}, 100 \mathrm{mg}$ and $200 \mathrm{mg}$ respectively for three groups. infants with neonatal cholestasis in three groups. Results: improvement of all of them, regarding liver histopathology wich the HCC focus regressed, regarding exosomal abundance in the serumand tissues while the evaluation of exosomal RAB11A-mRNA in rats' samples was performed by real time PCR, then the results were statistically analyzed by the SPSS software. Finally regarding to liver function test which the ALT, bilirubin and AFP reduced in the treated groups.
\end{abstract}

Conclusion: exosomal RAB11A-mRNA as biomarkers for HCC inducedrats and target these exosomal markers using Hesperidin in animal model and hence we can uses as thrapuetic bases in HCC in the future.

Keywords: HCC, Exosomes, DEN and 2-AAF and Hispiridine.

\section{INTRODUCTION}

Liver cancer is a leading cause of cancer deaths worldwide, accounting for more than 700,000 deaths each year The American Cancer Society's estimates of primary liver cancer and intrahepatic bile duct cancer in the United States for 2017 are about 40,710 of newly diagnosed cases and 28,920 died people of these cancers ${ }^{(\mathbf{1})}$. In Egypt; liver cancer is a serious if not the most serious cancer problem. It is ranked the first among cancers in males (33.6\%) and the third among females based upon results of National Cancer Registry Program (NCRP 2008-2011) ${ }^{(2)}$. The rising rates of hepatocellular carcinoma (HCC) in Egypt are due to the high prevalence of hepatitis B virus (HBV) and hepatitis $\mathrm{C}$ virus infection (HCV) among Egyptian population $^{(3)}$. Therefore; we need effective strategies for early detection and better management of HCC, which will be of great value in developing countries with limited resources and high incidence rates of HCC, such as Egypt. It has been well known that tumor development is a multistage process to accumulate alterations at the genetic and/or epigenetic level which ultimately reprogrammed a cell to undergo uncontrolled proliferation and metastasis (4). The expression of initial mutations depends not only on the internal interaction between oncogenes but also on external factors such as exosome, which could change the patterns of specific gene expression temporarily ${ }^{(5)}$.

Exosomes are cell-derived vesicles that convey key elements with the potential to modulate intercellular communication. They are known to be secreted from all types of cells, and are crucial messengers that can regulate cellular processes by 'trafficking' molecules from cells of one tissue to another ${ }^{(6)}$.

Exosomes originate when endocytic vesicles on the plasma membrane are fused to form early endosomes, and mature into late endosomes which undergo inward budding, in which vesicles form on the membrane of the endosome and are internalized ${ }^{(7)}$; At this point, the endosome contains multivesicular bodies (MVBs), while the vesicles within the MVB are called intraluminal endosomal vesicles (ILVs) . Rather than fusing with the lysosome, the MVBs fuse with the plasma membrane, releasing their internalized ILVs within extracellular space. Once the ILVs are released into extracellular space, they are considered exosome $^{(8)}$. Exosomes carry a variety of contents, including various proteins, DNA, mRNA, microRNA (miRNA), long non-coding RNA (lncRNA), and even viruses. According to the ExoCarta database (http://www.exocarta.org/), the presence of 41,860 proteins, more than 7540 RNA molecules and 1116 lipid molecules has been confirmed in exosomes in more than 286 studies ${ }^{(9)}$.

Exosomes are excreted by nearly all cells and contain genetic materials and various molecular signatures. they may be regarded as suitable biomarkers for cancer diagnosis, allowing for diagnosis and prognosis via a minimal invasive procedure. The transfer of exosomes between cells allows for intercellular communication an essential component for biological and pathobiological functions. Based on their functions, exosomes are considered molecular cargo ${ }^{(10)}$. Exosomes promote invasive phenotypes, 
angiogenesis and metastatic growth to the distant organs and, as a result, aid in therapeutics by acting as biomarkers and vehicles for genetic therapy; they can be potentially beneficial since exosomes are noninvasive bioavailable vehicles ${ }^{(11)}$.

On the other hand, the destruction/inactivation of cancer-derived exosomes itself may lead to inhibition of angiogenesis and metastasis, thus controlling the tumor. As a result, exosomes can be beneficial or destructive depending upon their environment. Their multi-faceted and dynamic role in cancer initiation, progression and metastasis offers a fertile ground to determine the cause, effect and treatment of various cancers. Ultimately, as research continues to decode the regulatory languages of exosomes' signals, this will pave the way for new strategies for cancer therapies ${ }^{(\mathbf{1 2})}$.

\section{AIM OF THE WORK}

1. To validate the expression of HCC-specific exosomal coding and non-coding RNAs after retrieving them from public microarray databases.

2. To characterize the efficacy of Hesperidin on modulating exosomal production and exosomal RNA related HCC expression using animal models.

\section{MATERIALS AND METHODS}

\section{A. Chemicals:}

Diethylnitrosamine (DEN), Acetamidofluorene (2-AAF), and urethane were purchased from Sigma Aldrich.

\section{B. Animals:}

This study included thirty male Wistar rats weighing 200-250g were used. All animal procedures were carried out in accordance with the National Institute of Health guide for the care and use of Laboratory animals (NIH Publication No. 85-23, revised 1996). Animals were housed in an animal room with temperature $\left(22-24^{\circ} \mathrm{C}\right)$ and lighting (12 h lightdark cycles). Rats received standard rat chow and tap water. An adaptation period of one week was allowed. The study was approved by the Ethics Board of AlAzhar University.

Experiments on animals were performed in Animal House of Faculty of Medicine, Ain-Shams University, according to the standard guidelines of care of laboratory animals. The animals were randomly selected and divided into the following:

Thirty rats were randomly divided into 5 groups (6 rats/each):

1. Control naïve group; rats were injected with $0.9 \%$ $\mathrm{NaCl}$.

2. $\mathrm{DEN}+2-\mathrm{AAF}$ group; rats were injected intraperitoneally with $100 \mathrm{mg} / \mathrm{kg}$ once weekly for 3 consecutive weeks followed by 1 -week rest period then $2 \mathrm{AAF}$ was injected once intraperitoneally in a dose of $300 \mathrm{mg} / \mathrm{kg}$ for induction of hepatic precancerous lesion.

3. Hesperidin 50mg group; rats were received DEN and $2 \mathrm{AAF}$ as mentioned above and treated with $50 \mathrm{mg} / \mathrm{kg}$ Hesperidin orally 4 times weekly, for 10 weeks (Mild-dose).
4. Hesperidin 100mg group; rats were received DEN and $2 \mathrm{AAF}$ as mentioned above and treated with $100 \mathrm{mg} / \mathrm{kg}$ Hesperidin orally 4 times weekly, for 10 weeks (moderate-dose).

5. Hesperidin 200mg group; rats were received DEN and $2 \mathrm{AAF}$ as mentioned above and treated with Hesperidin $200 \mathrm{mg}$ orally 4 times weekly, for 10 weeks (high- dose).

\section{Blood sample and liver tissue collection:}

At the end of the $10^{\text {th }}$ week rats were anesthetized with urethane $(1.2 \mathrm{~g} / \mathrm{kg}$; i.p.) dissolved in distilled water. Retro orbital blood samples were taken, then rats were sacrificed and liver sample were collected. For each animal, parts of the liver were kept in $-80{ }^{\circ} \mathrm{C}$ until analysis and other parts were kept in formaldehyde $10 \%$ for histopathology and immunohistochemistry staining examination.

D. Validation of the chosen biomarkers in animal model:

1. Exosomal RNA extraction.

2. Exosomes characterization.

3. Reverse transcription polymerase chain reaction (RT-PCR).

4. Quantitative Real Time Polymerase Chain Reaction (qPCR):

a. qPCR for detection of mRNA RAB11A.

b. qPCR for detection of Has-miRNA-1262.

c. qPCR for detection of LncRNA RP11-513I15.6

5. Set up amplification efficacy through standard curve, sensitivity and specificity of the assay.

6. Data collected and analysed.

\section{RESULTS}

The rats of the second group showed diffuse early and dysplastic nodule of HCC the normal hepatic architectures are distorted in some areas associated with fibrous tissue around foci, In some area appearance of lymphocytic infiltrations and congestion of portal and central veins as well as abnormality at the level of the cells (Figure 1). Masson trichrome stain show abundant of greenish color of fibrous tissue around the cancerous areas (figure 2).

Glutathione S Transferase placental form (GSTP) -Immuno-histochemical stain- revealed reaction of cancer cells in particular included wide range area of the affected tissues. (Figure 3). In the treated group the liver tissue regains nearly normal (Figure 4), and lower reaction of GSTP which stain cancer liver cell only (figure 5). On the TEM the exosomes were more abundant on the tissue with numerus mitochondria (Figure 6). There was a highly significant difference among the studied groups as regards fold change (RQ) of serum mRNA RAB11A, While comparing Hesperidin treated group and $\mathrm{HCC}$ group significant difference as regards fold change (RQ) of serum mRNA RAB11A in animal model tissues and serum.

In the luiver function it was observed that reduction off the liver function values in the treated group gradually to nearly normal specially on the high dose of hespiridine group. 


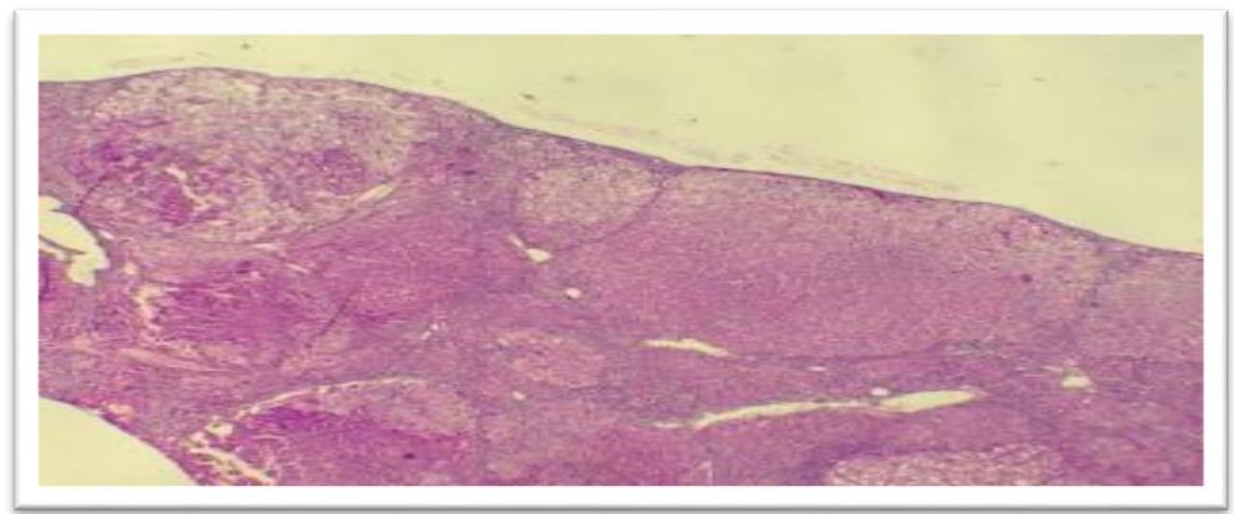

Figure (1): Liver tissue shows multiple diffuse foci of HCC 40 (H\&E).

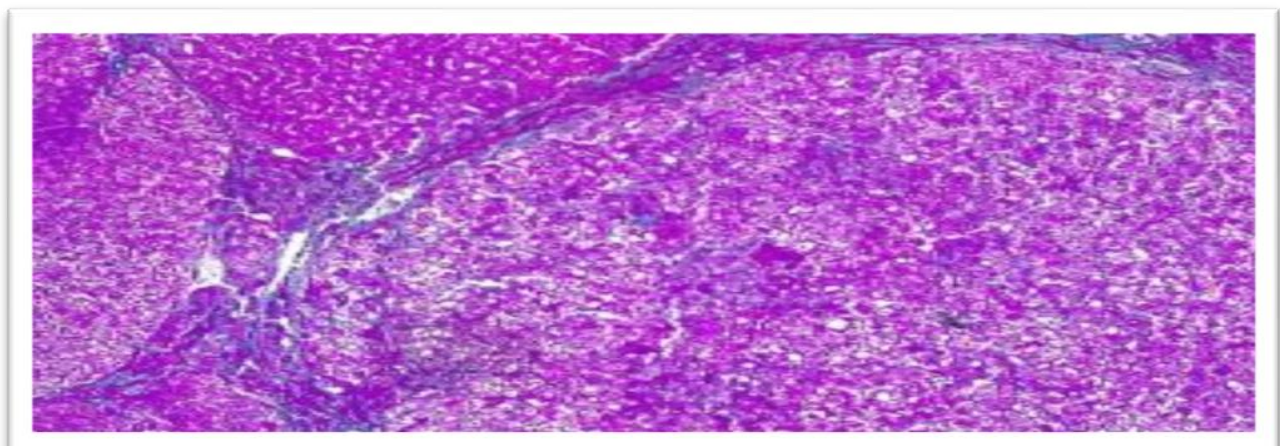

Figure (2): A photomicrograph showing green color of fibrous tissues surrounding the foci of HCC. X100 (Masson Trichrome stain)

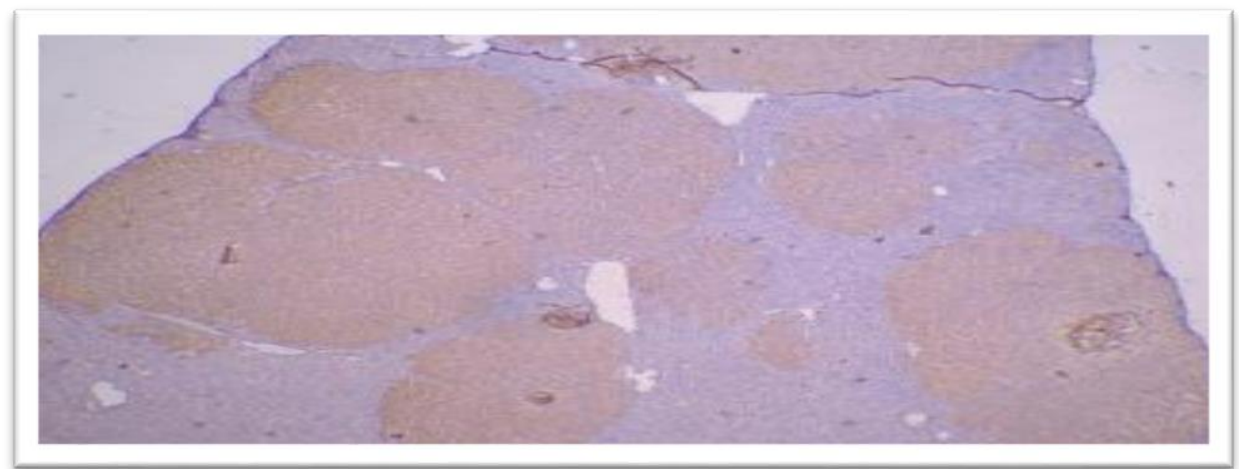

Figure (3): A photomicrograph showing brown discoloration reaction of cancer cell area X40 (GSTP)

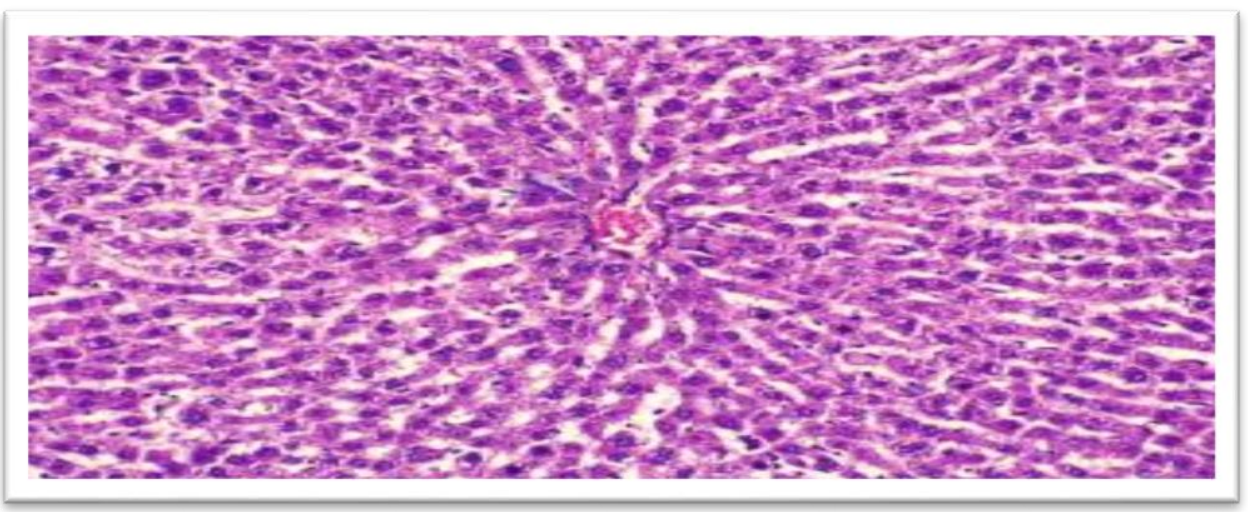

Figure (4): A micrograph of liver tissue showing no focus of HCC with more restoration of hepatic architecture nearly normal X200 H\&E 


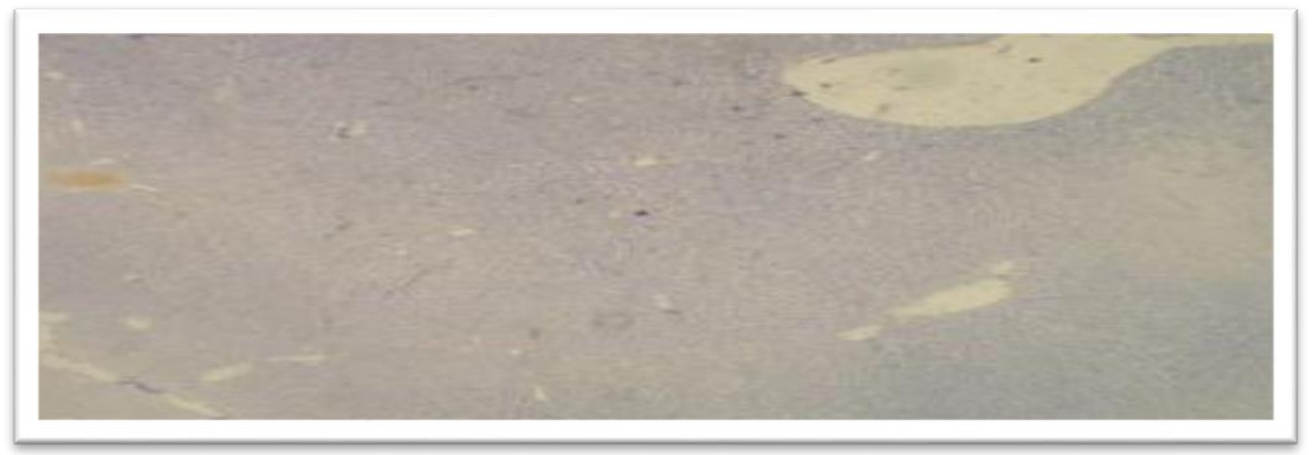

Figure (5): A micrograph of liver tissue showing no focus of HCC with more restoration of hepatic architecture nearly normal X40 (GSTP)

Table (1): Comparison between groups regarding to distribution of GSTP reaction of cancer cells area affected:

\begin{tabular}{|c|c|c|}
\hline & Stander Area & Affected Area \% \\
\hline Induced & 1920000 & $\mathbf{7 0 . 4 3 2}$ \\
\hline ttt mild dose & 1920000 & $\mathbf{5 2 . 2 5 1}$ \\
\hline ttt mod. Dose & 1920000 & $\mathbf{7 . 7 6 5}$ \\
\hline ttt high dose & 1920000 & $\mathbf{0 . 1 3 1}$ \\
\hline
\end{tabular}

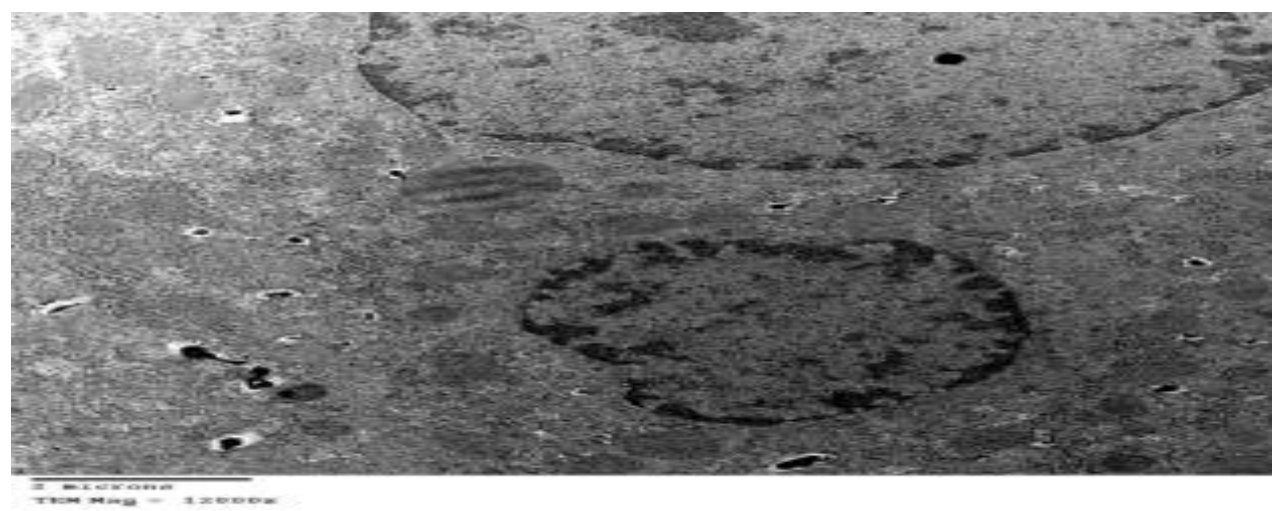

Figure (6): Electron micrographs of liver cancer cells showing numerus exosomes scattered with numerus mitochondria.

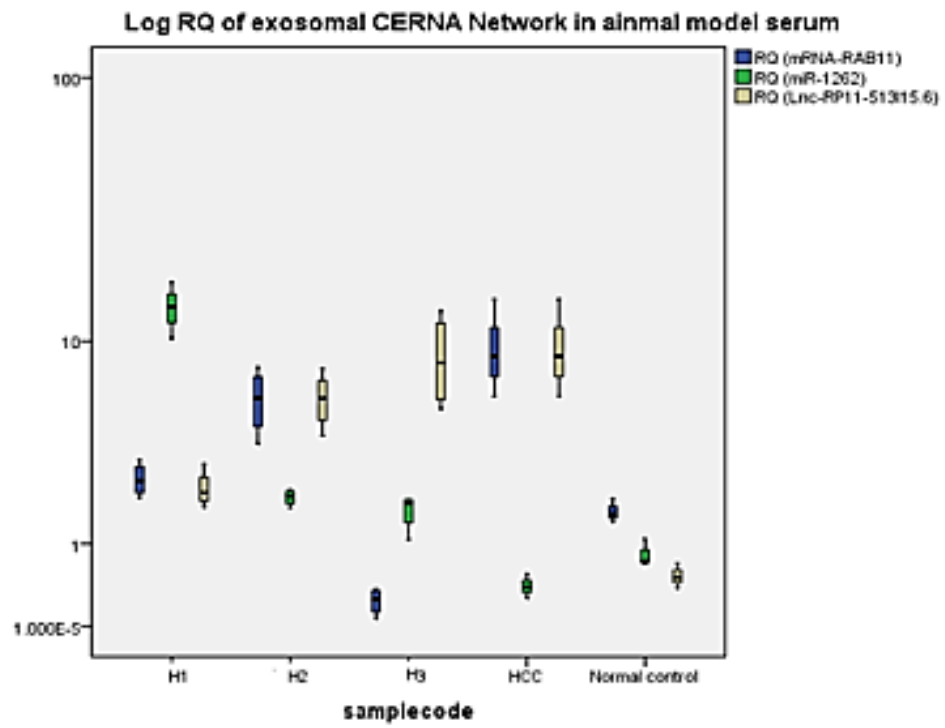

Figure (7): A graph showing the comparison between studied group regarding Log RQ of exosomal CERNA Network in animal model serum.

The results also showed that marked improvement on the liver tissues on the treated group and regression of the tumor affected area by using GSTP immunohistochemical stain. As well as the liver function (ALT, bilirubin \& AFP) improved markedly which observed reduced to nearly normal in treated groups. 


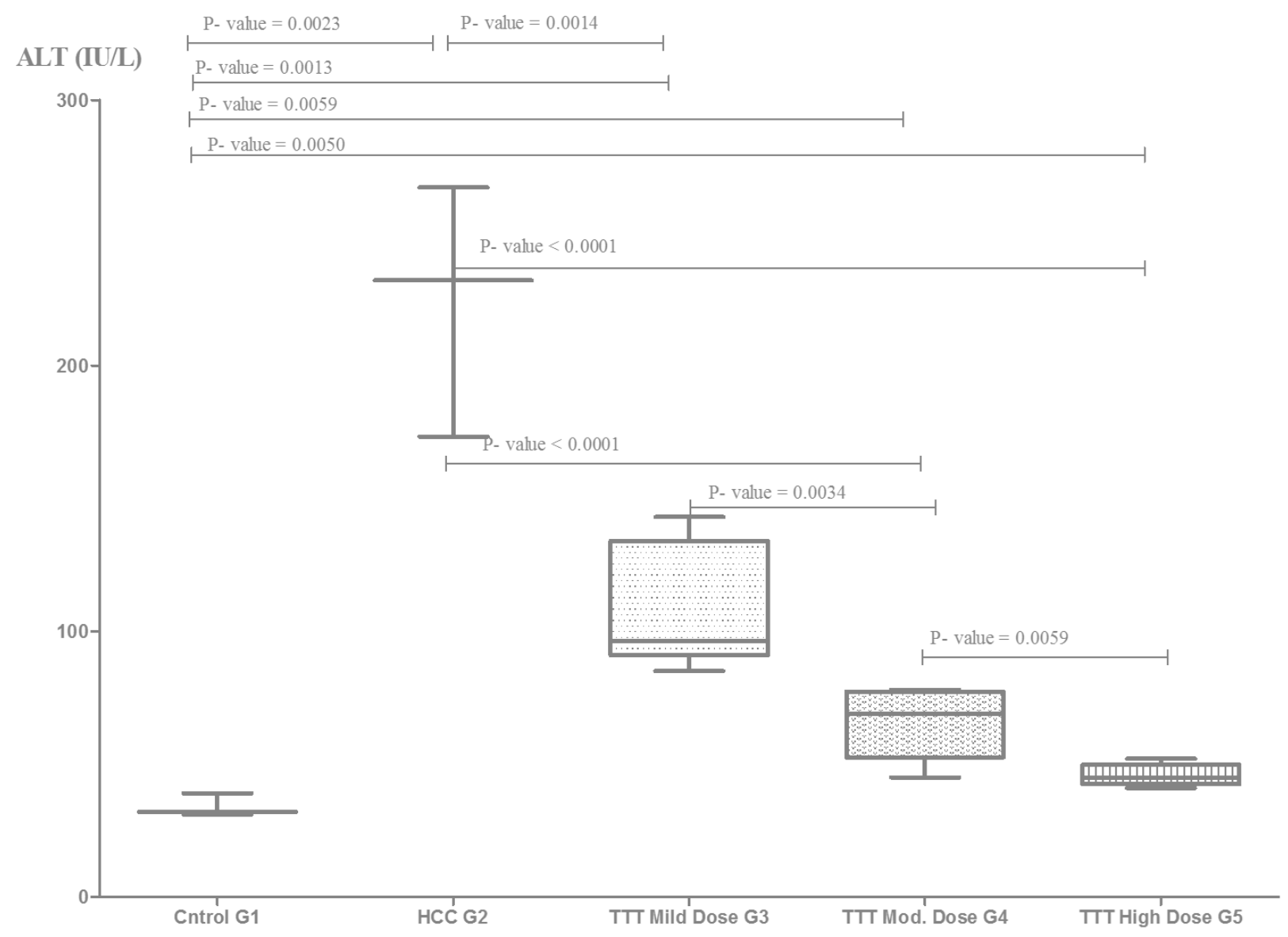

Figure (8): The graph shows differences between studied groups regarding ALT level which was high in HCC group and gradually reduced gradually in the treated groups contributing to the doses.

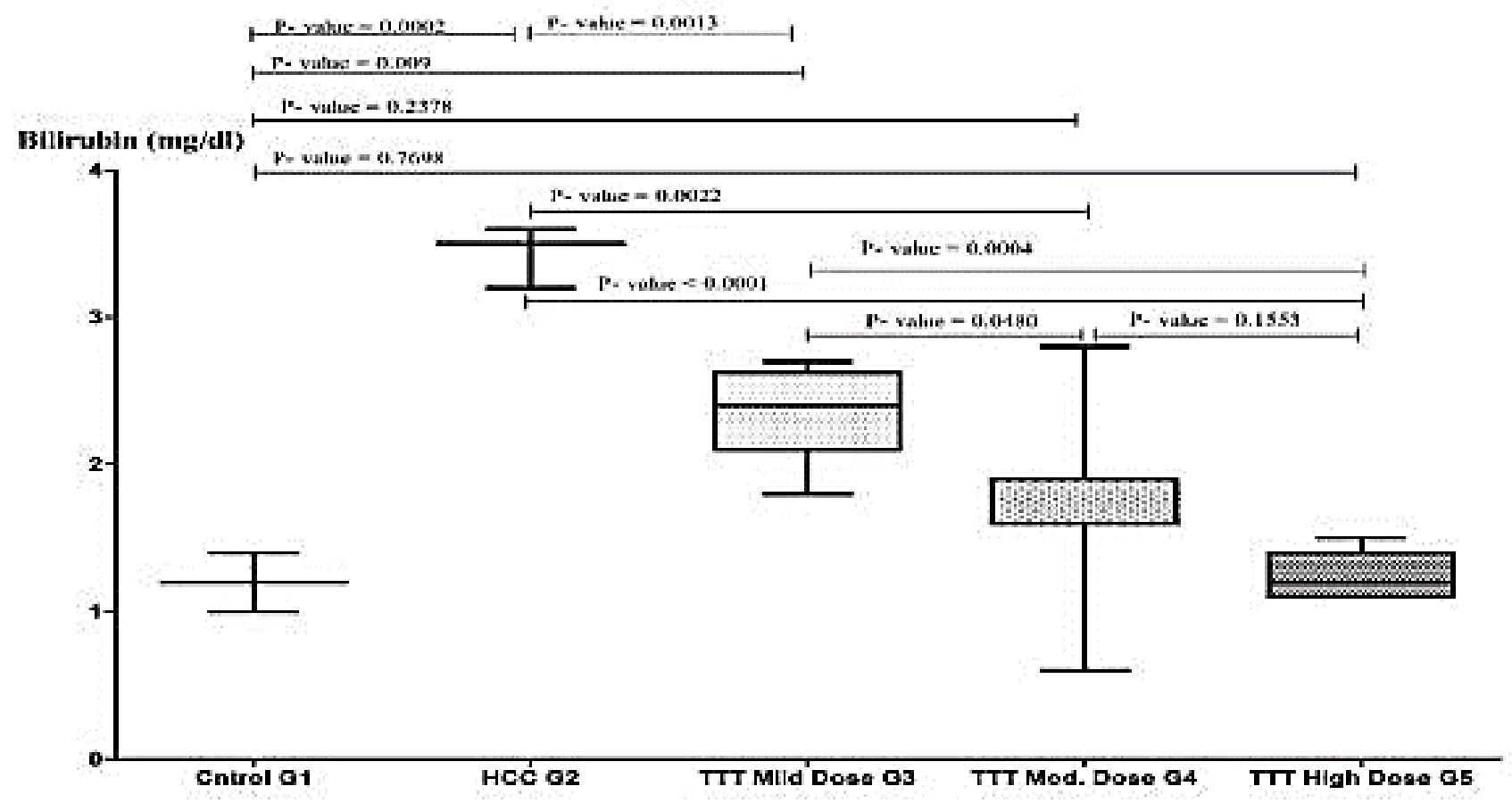

Figure (9): The graph shows differences between studied groups regarding bilirubin level which was high in HCC group and gradually reduced gradually in the treated groups regarding to the doses. 


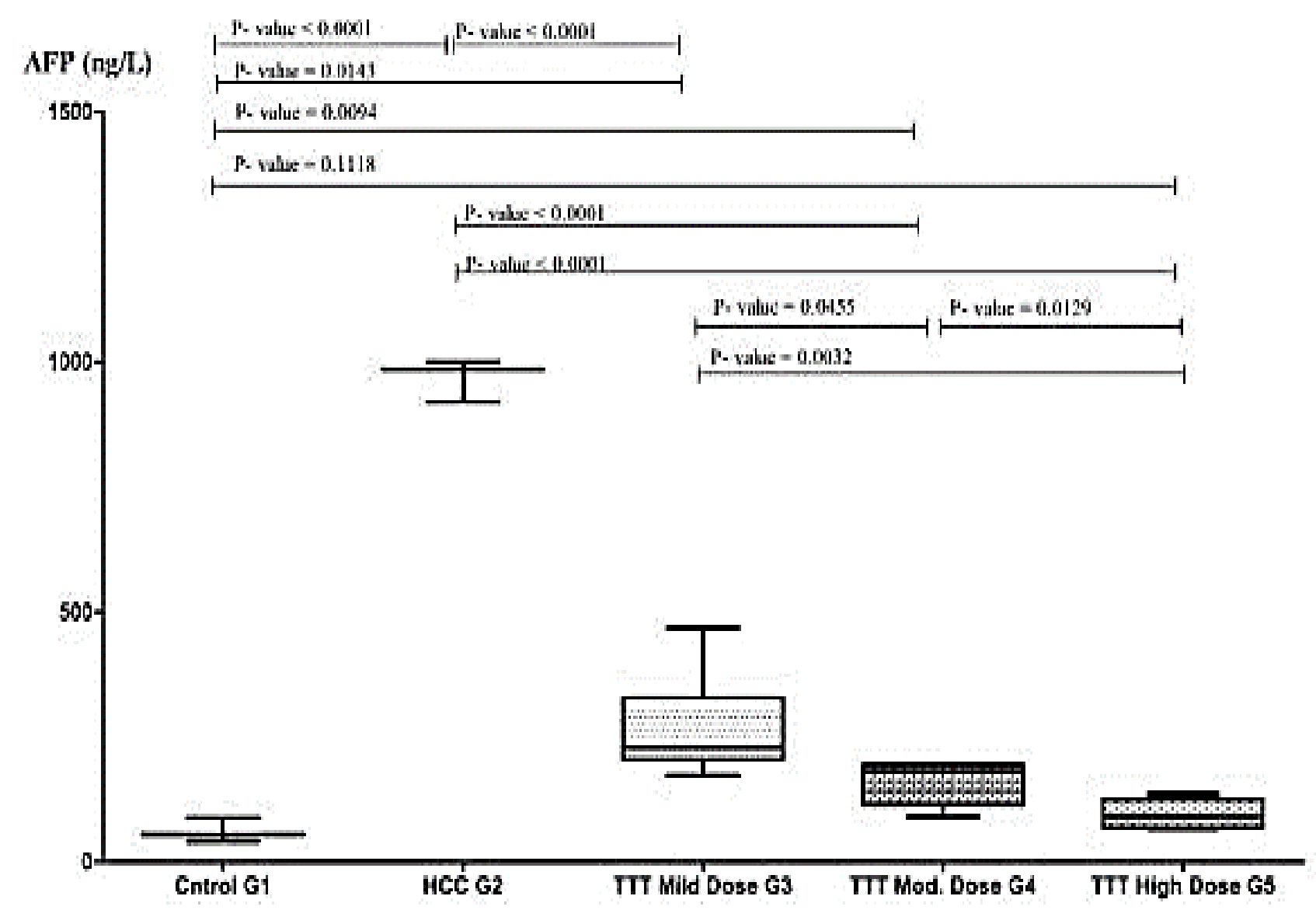

AFP = Alpha Feto Protein

Figure (10): The graph shows differences between studied groups regarding AFP level which was very high in HCC group and gradually reduced in the treated groups regarding to the doses.

\section{DISCUSSION}

In our study we used Hesperidin as novel therapeutic strategy for cancer, it is a part of flavonoids which are not only important natural antioxidants in the nature but also have a potent anticancer effects under both in vitro and in vivo conditions ${ }^{(\mathbf{1 3})}$.

As regarding to histology, noticed that the effect of Hesperidin administration on the tissue was abundant on the treated groups as regarding to the number and size of cells and affected area respectively, this was evident when we used in our study the GSTP immunohistochemical stain to measure the affected cancer-area, this reinforces that role of potential therapeutic effect of Hesperidin on the cancer ${ }^{(13,14)}$

Noteworthy the exosomes not only were abundance in circulation and exosomal related CERNA network but also were having a broader spread and more huge number on the EM images of affected tissue groups than treated one which informing that exosomes have a major role of spread of cancer ${ }^{(15)}$, thereby this point was the idea of our research, we found that exosomes not only enable to transfer genetic material to enhancing the cancer spread but also can be used as the therapeutic tole to suppress the effect of cancer activity.
While, newly discoveries identified the distinct non-coding RNAs were enriched in HCC exosomes, which strongly encouraged the idea of using these molecules as novel HCC biomarkers ${ }^{(16)}$.

Bioinformatics is applied for the exploration of cancer-related biomarkers, such as predisposition markers, diagnostics markers, prognostics markers, and therapeutics markers. Bioinformatics provides a sample base with access to clinical data for discovery, validation and evaluation of new biomarkers and drug targets ${ }^{(17)}$.

In light of these findings, we used integrative approach based on computational methods together with clinical validation to provide great insights into the molecular mechanisms of HCC. For the first time, we assessed a network of competing endogenous RNA (CERNA) involved in exosomal secretion which consists of RAB11A gene, retrieved from public databases. Afterwards, we investigated the chosen markers expression and diagnostic significance in $\mathrm{HCC}$ rats and control naïve groups. Then explore the effect of Hesperidin on this exosomal derived CERNA network.

In this study we analyzed $R A B 11 A$ gene expression which is important for exosomal secretion.

LncRNAs which are longer than 200 nucleotides are now known to have many functions, acting as 
scaffolds or guides to regulate interactions between protein and genes, as decoys to bind proteins or miRNAs, and as enhancers to modulate transcription of their targets (18). Moreover, lncRNAs possess significant regulatory effects on carcinogenesis and the development of cancer, demonstrating their potential roles in both oncogenic and tumorsuppressive pathways ${ }^{\left({ }^{(19)}\right.}$. Also some recent studies report a brand-new IncRNAs regulatory circuitry in which lncRNAs may function as competing endogenous RNAs (ceRNAs) and crosstalk with mRNAs by competitively binding their common miRNAs ${ }^{(20)}$.

The results of this study revealed that $R A B 11 A$ gene expression was up regulated in the liver tissues of HCC rat (median RQ 6.8) when compared to normal control rat (median RQ 0.71) and (mild, moderate and high dose) treated groups (median RQ $0.85,1.76$ and 0.42 ). there was significant difference among the studied groups as regards fold change (RQ) of $R A B 11 A$ gene expression in liver tissues $(\mathrm{P}<0.01)$.

The results of this study revealed that $R A B 11 A$ gene expression was up regulated in the serum of HCC rat (median RQ 8.7) when compared to normal control rat (median RQ 1.57) and (mild, moderate and high dose) treated groups (median RQ 2.41, 5.85 and 0.25). there was significant difference among the studied groups as regards fold change (RQ) of serum $R A B 11 A$ gene expression $(\mathrm{P}<0.01)$.

We presented a novel approach that enables a reliable integration of exosomal competing endogenous RNA network for HCC diagnosis. It is promising as a general strategy for future panel biomarker development in the serum of HCC patients and HCV patients. This can overcome the lower reliability of single-gene biomarker experiments and maintaining high accuracy by combining signals from multiple genetic levels. These findings expand the existing knowledge of RNA-RNA crosstalk characteristics and provide new tools to elucidate disease processes. Exosomes targeting by using Hesperidin to alter tumor microenvironment could offer new targets for HCC therapy.

Also, Previous reports done on tissue levels and cell lines indicate that $R A B 11 A$ serves as a cancer biomarker in pancreatic cancer ${ }^{(21)}$. Also, $R A B 11 A$ contributes to the malignant progression of non-small cell lung cancer NSCLC ${ }^{(22)}$.

It be thought that the exosomes execrated extensively from the cancer cells ${ }^{(23)}$, noted that on our research the exosomes not only expressed substantially on the tissue of the affected HCC group by TEM, but also significantly diminished on the treated groups 3,4 and 5, which strongly supported the idea of use exosomes based therapeutic notion.

As regarding to liver functions it was observed improvement on the different values (ALT, Bilirubin
\& AFP) on the treated group while the improvement was highly detected on the high dose group (5), and this supported with ${ }^{(24)}$. while he used the Hesperidin in the liver disease.

\section{CONCLUSION}

The exosomal RAB11A-mRNA can be considered as a marker for HCC as these markers showed marked differentiate between naïve, HCC and treated rats. Administration of Hesperidin alleviate these markers in $\mathrm{HCC}$ rats.

To the best of our knowledge this study is the first to measure the serum exosomal level of $R A B 11 A$ $m R N A$ in rats' serum and liver tissues of control, HCC and treated rats. Serum exosomal RAB11A-mRNA levels in serum sample is a potentially useful biomarker for early diagnosis and prediction of progression of HCC, Hesperidin could has benefit for HCC patients.

\section{REFERENCES}

1. American Cancer Society (2017): Cancer statistics, 2017. Cancer Journal for Clinicians, 67(1): 7-30.

2. Ibrahim AS, Hussein MK, Nabiel NHM et al. (2014):Cancer incidence in Egypt: results of the national population-based cancer registry program. Journal of cancer epidemiology.https://www.hindawi.com/journals/jce/ 2014/437971/

3. Abdelgawad IA, Mossallam G, Radwan NH et al. (2013): Can glypican3 be diagnostic for early hepatocellular carcinoma among Egyptian patients? Asian Pacific Journal of Cancer Prevention, 14(12): 7345-7349.

4. Gao Q, Zhao YJ, Wang XY et al. (2014): Activating mutations in PTPN3 promote cholangiocarcinoma cell proliferation and migration and are associated with tumor recurrence in patients. Gastroenterology, 146(5): 1397-1407.

5. Melo SA, Sugimoto H, O'Connell JT et al. (2014): Cancer exosomes perform cell-independent microRNA biogenesis and promote tumorigenesis. Cancer Cell, 26(5): 707-721.

6. Février $B$ and Raposo G (2004): Exosomes: endosomal-derived vesicles shipping extracellular messages. Current Opinion in Cell Biology, 16(4): 415-421.

7. Brinton LT, Sloane HS, Kester M et al. (2015): Formation and role of exosomes in cancer. Cellular and Molecular Life Sciences, 72(4): 659-671.

8. Lee Y, El Andaloussi S and Wood MJ (2012): Exosomes and microvesicles: extracellular vesicles for genetic information transfer and gene therapy. Human Molecular Genetics, 21(1): 125-134.

9. Keerthikumar S, Chisanga D, Ariyaratne D et al. (2016): ExoCarta: a web-based compendium of exosomal cargo. Journal of Molecular Biology, 428(4): 688-692. 
10.Kalluri R (2016): The biology and function of exosomes in cancer. The Journal of Clinical Investigation, 126(4): 1208-1215.

11. Tickner JA,Urquhart AJ, Stephenson SA et al. (2014): Functions and therapeutic roles of exosomes in cancer. Frontiers in Oncology, 4: 127-29.

12.Subramanian A (2016): Exosomes in carcinogenesis: molecular palkis carry signals for the regulation of cancer progression and metastasis. Journal of Cell Communication and Signaling, 10(3): 241-249.

13. Devi KP (2015): Hesperidin: A promising anticancer agent from nature. Industrial Crops and Products, 76: 582-589.

14. Ahmadi A (2015): The role of hesperidin in cell signal transduction pathway for the prevention or treatment of cancer. Current Medicinal Chemistry, 22(30): 34623471.

15. Azmi AS, Bao B and Sarkar FH (2013): Exosomes in cancer development, metastasis, and drug resistance: a comprehensive review. Cancer and Metastasis Reviews, 32(3-4): 623-642.

16. Takahashi K, Yan IK, Kogure T et al. (2014): Extracellular vesicle- mediated transfer of long noncoding RNA ROR modulates chemosensitivity in human hepatocellular cancer. FEBS open Bio., 4(1): 458-467.

17.Jain KK (2017): Technologies for Discovery of Biomarkers. In: The Handbook of Biomarkers, Springer., Pp. 27-96.
18.Li D, Liu X, Zhou J et al. (2017): Long noncoding RNA HULC modulates the phosphorylation of YB- 1 through serving as a scaffold of extracellular signalregulated kinase and YB- 1 to enhance hepatocarcinogenesis. Hepatology, 65(5): 1612-1627.

19.Du Z, Sun T, Hacisuleyman E et al. (2016): Integrative analyses reveal a long noncoding RNAmediated sponge regulatory network in prostate cancer. Nature Communications, 7: 10982-10986.

20. Karreth FA, Reschke M, Ruocco A et al. (2015): The BRAF pseudogene functions as a competitive endogenous RNA and induces lymphoma in vivo. Cell, 161(2): 319-332.

21. Yu L, Li X, Li H et al. (2016): Rab11a sustains GSK3 $\beta / W N T / \beta$-catenin signaling to enhance cancer progression in pancreatic cancer. Tumor Biology, 37(10): 13821-13829.

22.Dong Q, Fu L, Zhao Y et al. (2017): Rab11 a promotes proliferation and invasion through regulation of YAP in non-small cell lung cancer. Oncotarget, 8(17): 27800-27805.

23. Ohno SI, Takanashi M, Sudo K et al. (2013): Systemically injected exosomes targeted to EGFR deliver antitumor microRNA to breast cancer cells. Molecular Therapy, 21: 185-191.

24. Li C and Schluesener H (2017): Health-promoting effects of the citrus flavanone hesperidin. Critical Reviews in Food Science and Nutrition,57: 613-631. 